\title{
Limb Correction of Geostationary Infrared Imagery in Clear and Cloudy Regions to Improve Interpretation of RGB Composites for Real-Time Applications
}

\author{
NiCHOLAS J. ELMER \\ Department of Atmospheric Science, University of Alabama in Huntsville, and NASA Short-Term Prediction \\ Research and Transition Center, Huntsville, Alabama \\ EMILY BERNDT \\ Earth Science Office, NASA Marshall Space Flight Center, and NASA Short-Term Prediction Research and \\ Transition Center, Huntsville, Alabama \\ GARY JEDLOVEC \\ Earth Science Office, NASA Marshall Space Flight Center, Huntsville, Alabama \\ KEVIN FUELL \\ NASA Short-Term Prediction Research and Transition Center, and Earth and Space Science Center, University of \\ Alabama in Huntsville, Huntsville, Alabama
}

(Manuscript received 14 November 2018, in final form 26 June 2019)

\begin{abstract}
Red-green-blue (RGB) composites are increasingly used by operational forecasters to interpret vast amounts of satellite imagery by reducing several bands into a single, easily understood product which identifies important atmospheric features with unique colors. Limb effects, a result of an increase in optical pathlength of the absorbing atmosphere between the satellite and Earth as viewing zenith angle increases, adversely affects RGB composite interpretation by causing anomalous reductions in brightness temperature, thus changing the color interpretation of the RGB composites. In a previous paper, Elmer et al. demonstrated a limb correction technique that effectively removes limb effects from polar-orbiting infrared channels in both clear and cloudy regions using latitudinally and seasonally varying correction coefficients. This study applies the Elmer et al. limb correction to Air Mass RGB composites derived from geostationary sensors and compares the limb-corrected geostationary imagery to limb-corrected polar-orbiter imagery and satellite-derived atmospheric profiles. A statistical comparison in overlapping regions shows that the limb correction reduces the absolute mean brightness temperature difference from $4-12 \mathrm{~K}$ to $0-2 \mathrm{~K}$ for all infrared bands, demonstrating that the Elmer et al. limb correction algorithm is a robust method of removing limb effects from infrared imagery derived from both geostationary and polar-orbiting sensors. The limb-corrected RGB composites derived from geostationary sensors present several advantages, including the improved depiction of atmospheric features and enabling wider use of imagery from overlapping geostationary coverage regions where viewing zenith angles are large for both geostationary sensors.
\end{abstract}

\section{Introduction}

The development and launch of new multispectral sensors, starting with Meteorological Satellite (Meteosat) Second Generation (MSG) Spinning Enhanced Visible and Infrared Imager (SEVIRI; Schmetz et al. 2002) and followed by the Himawari-8 Advanced Himawari

Corresponding author: Nicholas J. Elmer, nicholas.j.elmer@ nasa.gov
Imager (AHI; Hidehiki et al. 2015), the Geostationary Operational Environmental Satellite 16 and 17 (GOES16/-17) Advanced Baseline Imager (ABI; Schmit et al. 2005; Lindsey et al. 2012), and the Meteosat Third Generation (MTG) Flexible Combined Imager (FCI; EUMETSAT 2018), allows for the creation of false-color multispectral composites, often called red-green-blue (RGB) composites. The European Organization for the Exploitation of Meteorological Satellites (EUMETSAT) designed the first RGB composite recipes for SEVIRI 

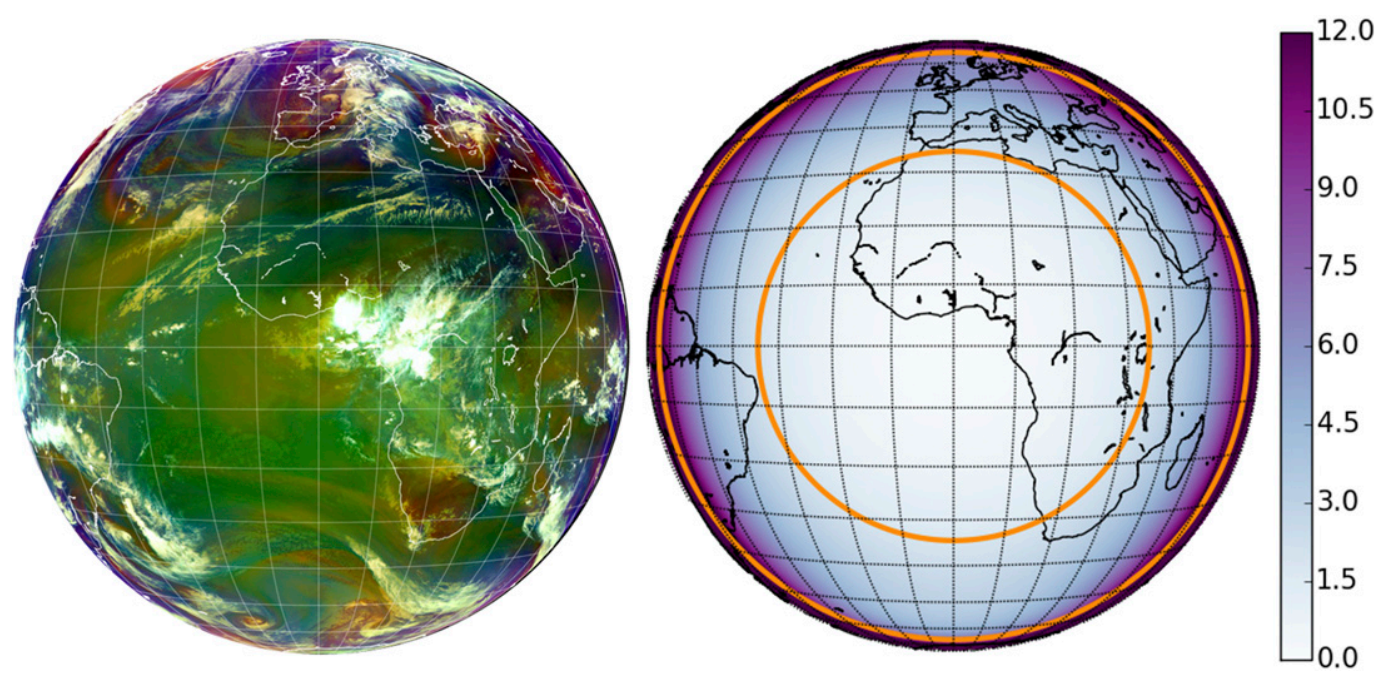

FIG. 1. (a) Evidence of limb cooling in the 1330 UTC 28 June 2015 SEVIRI Air Mass RGB is indicated by a reduction in green coloring (which also appears as an increase in blue or purple coloring) around the edge of the full disk image as the viewing zenith angle increases radially from the center of the image. (b) Brightness temperature difference (K) between limb-corrected and uncorrected SEVIRI band $6(7.3 \mu \mathrm{m})$ imagery used in (a). Viewing zenith angles of $40^{\circ}$ and $75^{\circ}$ are contoured in orange. Parallels and meridians are shown for every $10^{\circ}$ of latitude and longitude, respectively, with $\left(0^{\circ}, 0^{\circ}\right)$ in the center of the image.

to address specific forecast problems and developed a set of best practices for the future development and application of RGB composite recipes (Lensky and Rosenfeld 2008; EUMETSAT User Services 2009). The SEVIRI RGB recipes were successfully applied to AHI and $\mathrm{ABI}$ as well, requiring minor recipe adjustments to account for channel differences between sensors (Elmer et al. 2016; Berndt et al. 2018). RGB composites typically combine thermal infrared and/or solar channels into a single composite image to highlight important atmospheric features. For example, the Air Mass RGB (EUMETSAT 2015a; Lensky and Rosenfeld 2008 ), which assists in the detection of potential vorticity anomalies, rapid cyclogenesis, frontal systems, and upper-air water vapor content (Zavodsky et al. 2013; Berndt et al. 2016) combines the 6.2-, 7.3-, 9.6-, and $10.8-\mu \mathrm{m}$ bands (see Fig. 1a and Table 1). The 6.2- and 7.3- $\mu \mathrm{m}$ bands are considered water vapor channels, since longwave radiation emitted at these wavelengths are significantly absorbed by atmospheric water vapor. The $9.6-\mu \mathrm{m}$ band provides information about atmospheric ozone, as it lies in an ozone absorption band. Last, the $10.8-\mu \mathrm{m}$ band lies in the atmospheric window, and therefore experiences little atmospheric absorption, thus providing an estimate of surface temperature on cloud-free areas and cloud-top temperature if thick clouds are present. The red component of the Air Mass RGB, made up of the 6.2- minus 7.3- $\mu \mathrm{m}$ brightness temperature difference, indicates the vertical gradient of water vapor, where a large red contribution implies dry upper levels. The green component, composed of the 9.6- minus $10.8-\mu \mathrm{m}$ brightness temperature difference, qualitatively estimates tropopause height based on total ozone, where a large green contribution indicates ozone-poor, tropical air masses with a high tropopause height and a low green contribution indicates ozone-rich, polar air masses with low tropopause height. The blue component, which is simply the inverted $6.2-\mu \mathrm{m}$ brightness temperature, indicates upperlevel moisture, where a large blue component indicates moist upper levels or high clouds (EUMETSAT 2015a; Zavodsky et al. 2013; Lensky and Rosenfeld 2008). Combining these color components, the resulting Air Mass RGB identifies potential vorticity (PV) anomalies, jet streaks, stratospheric intrusions, and regions of large-scale subsidence as red; cold, ozonerich air masses as blue and purple; warm air masses with high, upper-tropospheric humidity as green; and warm air masses with low, upper-tropospheric humidity as olive (EUMETSAT 2015a; Lensky and Rosenfeld 2008). A more detailed description of the Air Mass

TABLE 1. EUMETSAT SEVIRI Air Mass RGB recipe from EUMETSAT (2015a). Note that no gamma correction is applied to any of the channels or channel differences.

\begin{tabular}{lcc}
\hline \hline Color & Channel (difference) & Range \\
\hline Red & $6.2-7.3 \mu \mathrm{m}$ & -25 to $0 \mathrm{~K}$ \\
Green & $9.7-10.8 \mu \mathrm{m}$ & -40 to $5 \mathrm{~K}$ \\
Blue & $6.2 \mu \mathrm{m}$ inverted & 243 to $208 \mathrm{~K}$ \\
\hline
\end{tabular}


RGB and several other common RGB composites can be found in Lensky and Rosenfeld (2008), Fuell et al. (2016), Zavodsky et al. (2013), and Berndt et al. (2018).

Infrared imagery is adversely affected by limb effects, or limb cooling, which is the result of an increase in optical pathlength of the absorbing atmosphere by trace gases (e.g., water vapor, ozone, and carbon dioxide) as satellite viewing zenith angle increases (Soden and Bretherton 1993; Goldberg et al. 2001; Elmer et al. 2016). Limb cooling is primarily known to affect infrared imagery derived from nadir-scanning polar-orbiting sensors, since the optical pathlength increases rapidly over relatively short ground distances of less than a few hundred kilometers for these sensors, distorting the coloration of mesoscale and synopticscale features in the RGB composites. RGB composites derived from geostationary sensors are also affected by limb effects but to a lesser extent, since viewing zenith angles change slowly over large distances. However, this still adversely impacts RGB interpretation on the limb, since air masses are not shown with their appropriate RGB coloration, as shown by Fig. 1 for a SEVIRI Air Mass RGB. Note that the reduction in green coloring near the edge of the full disk image (Fig. 1a), which indicates that brightness temperatures in the $9.6-\mu \mathrm{m}$ band are significantly reduced by limb cooling (i.e., increased absorption by atmospheric ozone due to a greater depth of atmosphere being viewed), resulting in an RGB coloration that does not represent atmospheric conditions. Since the optical pathlength varies little from nadir to viewing zenith angles of approximately $40^{\circ}$ (Fig. 1b), limb effects are minimal $(<2 \mathrm{~K})$, so interpretation of the RGB is relatively unaffected in this region. However, limb effects can adversely affect interpretation for viewing zenith angles of $40^{\circ}-75^{\circ}$. For viewing zenith angles greater than $75^{\circ}$, the limb effect is substantial.

Elmer et al. (2016) developed a limb correction algorithm that removes limb effects from the Moderate Resolution Imaging Spectroradiometer (MODIS) and Suomi National Polar-Orbiting Partnership (SNPP) Visible Infrared Imaging Radiometer Suite (VIIRS) infrared channels in both clear and cloudy regions using latitudinally and seasonally varying limb correction coefficients. The limb correction equation [Elmer et al. 2016, their Eq. (6)] is given as

$$
\begin{aligned}
T_{\mathrm{CORR}}= & T_{B}-T_{\mathrm{OFFSET}}+Q\left[C_{2}(\varphi, \delta) \ln \left(\cos \theta_{z}\right)^{2}\right. \\
& \left.-C_{1}(\varphi, \delta) \ln \left(\cos \theta_{z}\right)\right],
\end{aligned}
$$

where $T_{\mathrm{CORR}}$ is the limb-corrected brightness temperature, $T_{B}$ is the sensor-measured brightness temperature, $T_{\text {OFFSET }}$ is a constant value specific to a sensor band that corrects for channel differences between sensors (see Elmer et al. 2016), and $\theta_{z}$ is the satellite viewing zenith angle. The cloud correction factor $Q$ is a function of cloud-free atmospheric transmittance and ranges between 0.0 (cloud detected at or above the tropopause) and 1.0 (cloud detected at the surface or no cloud detected) (Elmer et al. 2016). Note that when no cloud is present $(Q=1)$, the full limb correction is performed, whereas when a very high cloud is present, the last term of Eq. (1) approaches zero and little to no limb correction is performed. Note that cloud movement, parallax, or observing the sides of clouds due to large viewing angles are not addressed by the Elmer et al. (2016) limb correction algorithm. The implications of this are described further in section 3. In the square brackets, $C_{1}(\varphi, \delta)$ and $C_{2}(\varphi, \delta)$ are the channel specific limb correction coefficients as a function of latitude $\varphi$ and Julian day $\delta$, based on a quadratic best-fit described by

$$
T_{\theta_{z}}-T_{0}=C_{2}\left|\ln \left(\cos \theta_{z}\right)\right|^{2}+C_{1}\left|\ln \left(\cos \theta_{z}\right)\right|
$$

where $T_{0}$ and $T_{\theta_{z}}$ are brightness temperatures derived from the Joint Center for Satellite Data Assimilation (JCSDA) Community Radiative Transfer Model (CRTM; Han et al. 2006) at nadir and $\theta_{z}$ using a large sample of model atmospheric profiles. The values of $C_{2}$ range from 0 for water vapor and ozone channels to approximately 2 for window channels, whereas $C_{1}$ ranges from 0 for window channels to approximately 8-12 for water vapor and ozone channels (Elmer et al. 2016).

To apply the limb correction, the required inputs are brightness temperature, viewing zenith angle, latitude, and cloud-top pressure for each pixel. It is difficult to implement this approach on geostationary imagery for viewing zenith angles greater than $75^{\circ}$ due to the sensors measuring minimal surface contribution at very low spatial resolution and inaccurate cloud-top pressure calculations. Elmer et al. (2016) showed that the limb correction of MODIS and VIIRS infrared channels led to improved RGB composites, allowing for accurate interpretation by operational forecasters. The research described in this paper extends Elmer et al. (2016) to demonstrate that the same limb correction algorithm can be applied to SEVIRI, AHI, ABI, and FCI infrared imagery in order to create limb-corrected Air Mass RGB composites. 


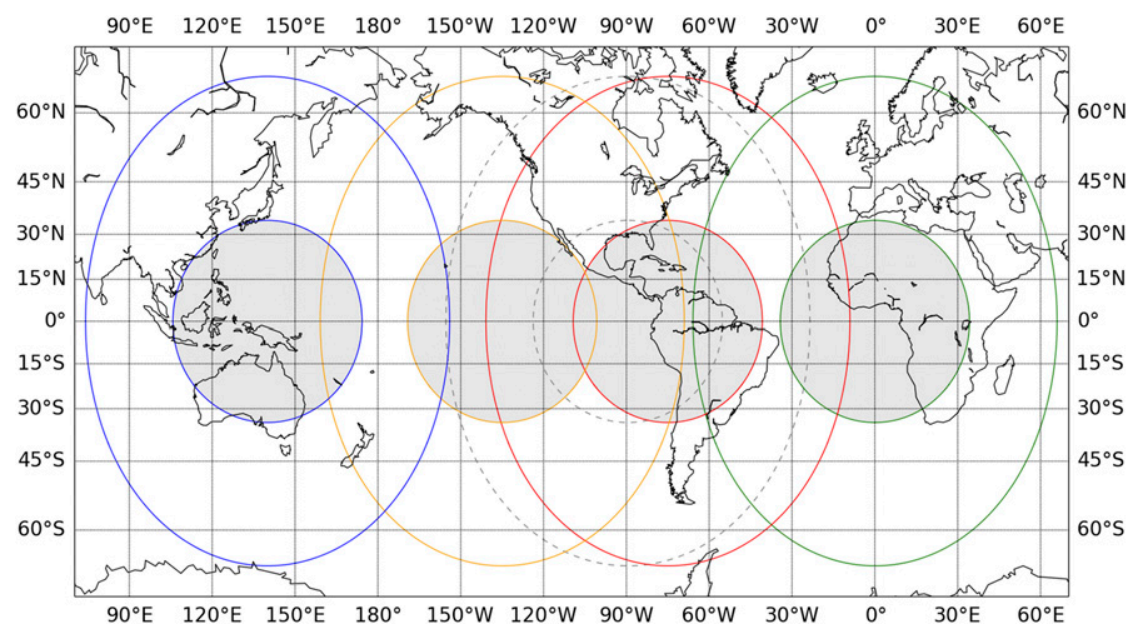

FIG. 2. Coverage of AHI (blue), GOES-17/West ABI (orange), GOES-16/East ABI (red), and SEVIRI (green), with nadir longitudes of $140^{\circ} \mathrm{E}, 135^{\circ} \mathrm{W}, 75^{\circ} \mathrm{W}$, and $0^{\circ}$, respectively. Shaded regions indicate viewing zenith angles less than $40^{\circ}$ where limb effects are minimal, whereas the unshaded regions indicate viewing zenith angles of $40^{\circ}-75^{\circ}$ where limb effects are significant but can be corrected using the Elmer et al. (2016) limb correction algorithm. The gray dashed lines indicate the coverage of $G O E S$-16/East at the time of the case study included in this paper with a nadir longitude of $89.5^{\circ} \mathrm{W}$.

\section{Data and methodology}

This study uses satellite imagery from the Meteosat10 SEVIRI, Himawari-8 AHI, and GOES-16 ABI sensors, which have similar channels, to create Air Mass RGB imagery. The coverage of each of these sensors is shown in Fig. 2, which also includes the GOES-17 ABI field of view. Meteosat-10 SEVIRI full disk level 1.5 image, geolocation, and cloud data were obtained from the EUMETSAT Data Centre (EUMETSAT 2015b) and Himawari-8 AHI full disk data were retrieved from the National Environmental Satellite, Data, and Information Service (NESDIS) Center for Satellite Applications and Research (STAR) provided by the Japan Meteorological Agency (JMA). GOES-16 ABI full disk data were obtained directly from the NASA Marshall Space Flight Center GOES-16 receiving station. A total of four case studies were selected to demonstrate the accuracy of the limb correction methodology for several different geostationary sensors and over a variety of conditions (Table 2), and are intended to demonstrate the broad applicability of the limb correction algorithm. Note that this limb correction is not objectively being tested for quantitative accuracy, but rather whether it is qualitatively effective for operational forecasting, because qualitative improvements that assist with RGB interpretation are still beneficial to forecasters.

The infrared imagery from SEVIRI, AHI, and ABI were limb-corrected following the Elmer et al. (2016) methodology, and the limb-corrected bands were used to create Air Mass RGB composites. Although many other RGB recipes exist, only the Air Mass RGB is used in case studies shown in this paper, since the Air Mass RGB incorporates three bands highly sensitive to water vapor and ozone and thus is more strongly impacted by limb effects than the other RGB products. Limb-corrected Air Mass RGB composites were also created from Aqua MODIS swaths corresponding to the case studies for qualitative comparison with respect to the geostationary limb correction. Since Aqua MODIS is a polar-orbiting sensor, nadir points run the

TABLE 2. Four case studies selected to demonstrate the limb correction for multiple geostationary sensors with variable seasons and times of day.

\begin{tabular}{lcccc}
\hline \hline Figure & Geostationary sensor & Reference sensor & Time & Date \\
\hline Fig. 3 & MSG SEVIRI & Aqua MODIS & 1330 UTC (day) & 28 Jun 2015 \\
Fig. 4 & MSG SEVIRI & Aqua MODIS & 0945 UTC (day) & 5 Mar 2016 \\
Fig. 6 & Himawari-8 AHI & Aqua MODIS & 1630 UTC (night) & 21 Oct 2015 \\
Fig. 9 & GOES-16 ABI & MSG SEVIRI & 0000 UTC (night) & 6 Sep 2017 \\
\hline
\end{tabular}


entire length of the swath, enabling comparison of the limb-corrected geostationary imagery on the limb at points that are unaffected by limb effects for MODIS. Aqua MODIS (Collection 6 and 6.1) 1-km 5-min level 1B calibrated radiances (MYD021KM), $1 \mathrm{~km}$ geolocation (MYD03), and 1-km 5-min level 2 cloud product (MYD06_L2) were retrieved from the Level 1 and Atmosphere Archive and Distribution System (LAADS; GSFC 2015). Aqua Atmospheric Infrared Sounder (AIRS; Aumann et al. 2003) and SNPP Cross-track Infrared Sounder/Advanced Technology Microwave Sounder (CrIS/ATMS) NOAA Unique Combined Atmospheric Processing System (NUCAPS; Gambacorta 2013; Gambacorta and Barnet 2013) atmospheric profiles were also obtained for comparison to ensure the limb-corrected RGB composite color interpretation matched the observed atmospheric conditions. Although NUCAPS soundings will be smoother than those that could be obtained from radiosondes, they have been extensively validated against reference radiosondes with stringent space-time collocation criteria (Nalli et al. 2018). NUCAPS satellite-derived soundings can retrieve moisture and temperature in the mid- and upper levels with temperature profile uncertainties near $1 \mathrm{~K}$ and moisture profile uncertainty ranging from 0.02 to $0.30 \mathrm{~g} \mathrm{~kg}^{-1}$ (Nalli et al. 2018) and are suitable for assessing a varied range of moisture transport regimes (Nalli et al. 2016).

With the advent of advanced sensors with more precise spectral characteristics, there is a need to account for differences in the spectral response function when deriving and comparing RGB imagery from different sensors. Small differences in brightness temperature or reflectance due to differences in spectral characteristics can impact RGB coloring and interpretation across sensors. The best practices EUMETSAT RGB recipes were developed with SEVIRI, which has wider spectral bandwidths than many of the research and next generation sensors (MODIS, VIIRS, AHI, ABI, FCI). For example, the SEVIRI 3.9- $\mu \mathrm{m}$ band includes a region of the electromagnetic spectrum with carbon dioxide absorption. Therefore when deriving RGB imagery that depends on the 3.9- $\mu \mathrm{m}$ band (e.g., Nighttime Microphysics RGB), with sensors such as ABI with a narrower spectral bandwidth, the resulting image does not match the standard RGB coloring and interpretation. Therefore, to create consistent RGB imagery across legacy and next-generation sensors, one must account for spectral differences across sensors through applying a $T_{\text {OFFSET }}$ (Elmer et al. 2016) or adjusting the RGB recipe thresholds (EUMETSAT User Services 2009; Shimizu 2015; Zeschke 2015; Kerkmann 2016;
Scarino et al. 2016; Berndt et al. 2018). To create consistent Air Mass RGBs between MODIS, SEVIRI, AHI, and ABI for intercomparison, the EUMETSAT SEVIRI Air Mass RGB recipe was adjusted following the Berndt et al. (2018) intercalibration and recipe adjustment methodology prior to applying the RGB recipe to MODIS, AHI, and ABI infrared channels. This method is similar to the method of Shimizu (2015) as demonstrated in Zeschke (2015) and Kerkmann (2016). Note that while Elmer et al. (2016) accounts for channel differences between sensors using of the $T_{\text {OFFSET }}$ term in Eq. (1), Berndt et al. (2018) removes this term from the limb correction equation and instead modifies the SEVIRI RGB recipe based on linear regression to create a sensor-unique RGB recipe (e.g., ABI RGB recipe). However, either method is able to account for differences in the spectral response function between compared sensors to achieve identical limb-corrected RGB products. Therefore, since the Berndt et al. (2018) recipe adjustment methodology already accounts for channel differences between sensors, the $T_{\text {OFFSET }}$ term in Eq. (1) was neglected by this study during limb correction.

For figures within this paper showing Aqua MODIS imagery overlaying geostationary imagery, the MODIS imagery was resampled to match the geostationary projection and resolution. For the figures comparing two geostationary sensor RGBs, the imagery was resampled to match the geostationary sensor with the highest spatial resolution. For reference, SEVIRI has a nominal resolution of $3 \mathrm{~km}$, while $\mathrm{AHI}$ and $\mathrm{ABI}$ have a nominal resolution of $2 \mathrm{~km}$. MODIS has a nominal resolution of $1 \mathrm{~km}$.

\section{Results and discussion}

a. 1330 UTC 28 June 2015

The first case study compares the 1330 UTC 28 June 2015 SEVIRI Air Mass RGB against the corresponding Aqua MODIS Air Mass RGB swath (points with viewing zenith angles less than $40^{\circ}$ only) that passes through the center of the SEVIRI field of view, such that Aqua MODIS and SEVIRI have shared nearnadir points near the equator (Fig. 3). Aqua MODIS also passes directly over the north and south limbs of the SEVIRI field of view in the same pass, so that the Aqua MODIS swath, which requires minimal limb correction, can be directly compared to the limbcorrected northern and southern limbs of the SEVIRI Air Mass RGB. The color tile pairs in Fig. 3 compare the uncorrected and limb-corrected coloration of the SEVIRI Air Mass RGB to the limb-corrected 

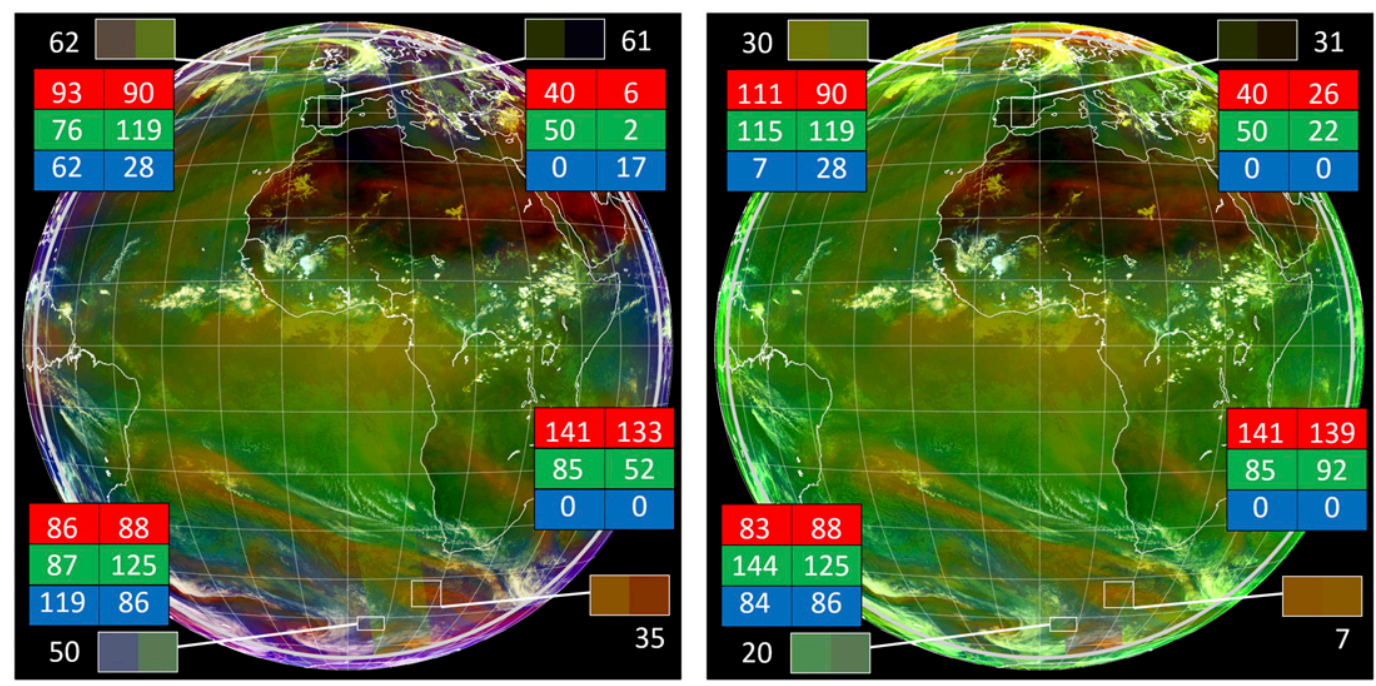

FIG. 3. The 1330 UTC 28 June 2015 limb-corrected Aqua MODIS Air Mass RGB (viewing zenith angles $<40^{\circ}$ only) merged with (a) uncorrected and (b) limb-corrected SEVIRI Air Mass RGB. The color tiles depict the RGB color of each tile pair for SEVIRI (outside color tile) and MODIS (inside color tile). The corresponding RGB triplets (which can range from 0 to 255 for each color component) are shown in the table next to each color tile pair with the associated Euclidean distance in RGB space between the SEVIRI and Aqua MODIS color tiles for each tile pair.

Aqua MODIS Air Mass RGB. The RGB triplets, which shows the red, green, and blue contributions for a given color, are shown for each color tile pair, along with the distance between the two colors in RGB space. Similar to calculating the Euclidean distance in Cartesian coordinates, the RGB distance is given by

$$
D=\sqrt{\left(R_{\mathrm{SEVIRI}}-R_{\mathrm{MODIS}}\right)^{2}+\left(G_{\mathrm{SEVIRI}}-G_{\mathrm{MODIS}}\right)^{2}+\left(B_{\mathrm{SEVIRI}}-B_{\mathrm{MODIS}}\right)^{2}},
$$

where $R, G$, and $B$ are the red, green, and blue byte values, respectively, which range from 0 to 255 . Thus, as colors become more similar, the RGB distance will decrease.

In Fig. 3a, the uncorrected SEVIRI Air Mass RGB are clearly impacted by limb effects as viewing zenith angles increase. Although both present visually similar coloring at nadir where limb effects are minimal, the SEVIRI Air Mass RGB shows noticeably different coloring than the Aqua MODIS Air Mass RGB moving toward the limb. The color tile pairs show considerable differences in the red, green, and blue components, with RGB distances ranging from 35 to 62 . Figure 3b compares the limb-corrected Aqua MODIS Air Mass RGB against the limb-corrected SEVIRI Air Mass RGB, where the Aqua MODIS swath blends into the underlying SEVIRI RGB, clearly indicating that the limb correction enables more consistent interpretation throughout both the Aqua MODIS and SEVIRI Air Mass RGB composites (Fig. 3b). The color tile pairs now depict similar coloring between the
SEVIRI and MODIS Air Mass RGBs. The RGB triplets and the decreasing RGB distances, which now range from 7 to 31, confirm that the SEVIRI and Aqua MODIS RGBs are more consistent following limb correction. Remaining discrepancies between the RGB triplets and color tiles are attributed to subtle variations created by using the adjusted RGB recipes to account for channel differences between sensors, uncertainty in the limb correction coefficient derivation (Elmer et al. 2016), and movement of atmospheric features due to imagery time difference. For Fig. 3, the time difference between nadir samples is less than $1 \mathrm{~min}$ with both occurring near 1308 UTC. At Southern and Northern Hemisphere high latitudes, the time difference is approximately 20 and $12 \mathrm{~min}$, respectively. For some color tile pairs, the red component difference sometimes increases, rather than decreases, with limb correction, which makes it appear like the limb correction degrades the RGB. However, this is a result of the Air Mass RGB recipe, which differences the 6.7- and $7.3-\mu \mathrm{m}$ bands. The magnitude of limb cooling in these 

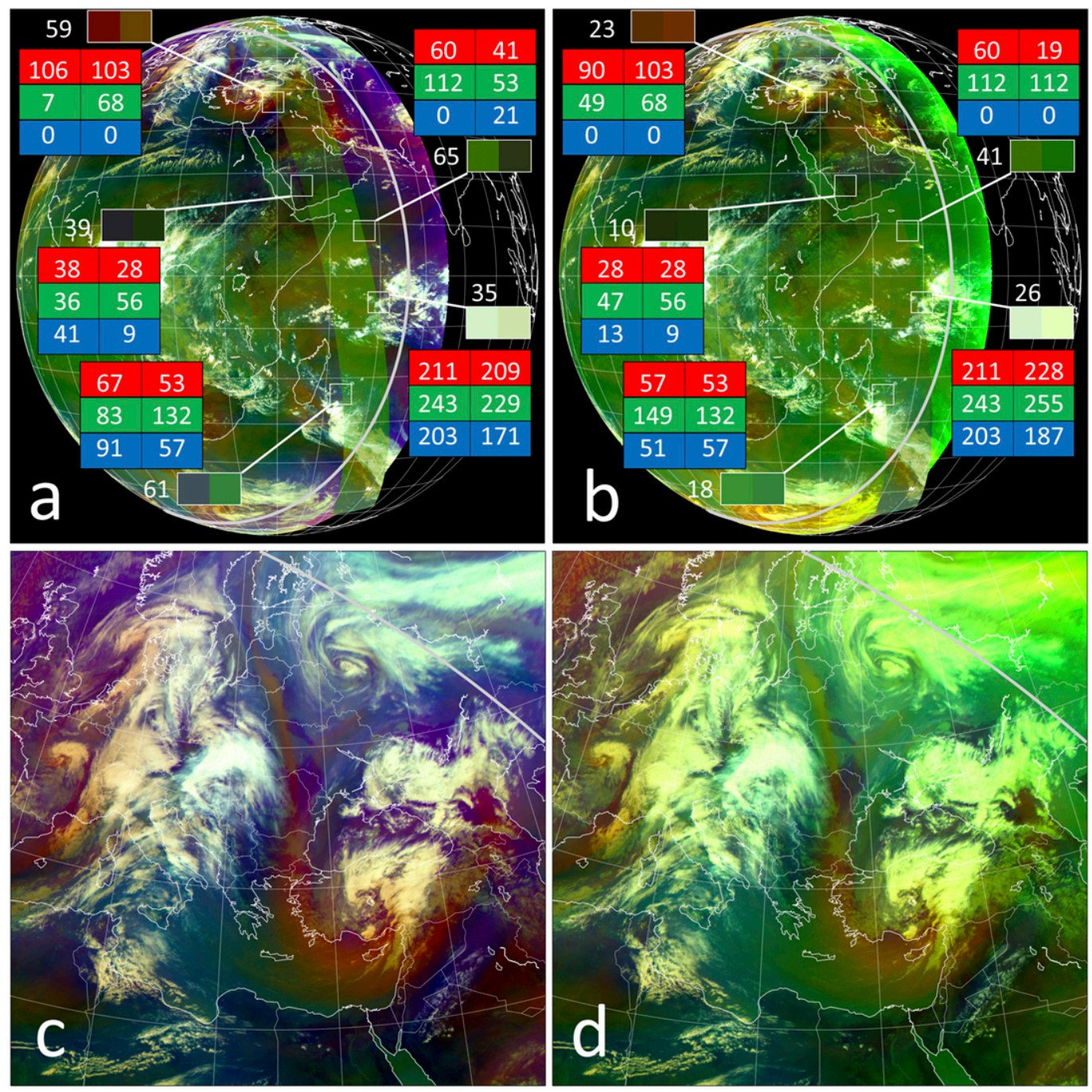

FIG. 4. The 1000 UTC 5 March 2016 limb-corrected Aqua MODIS Air Mass RGB (viewing zenith angles $<40^{\circ}$ only) merged with (a) uncorrected and (b) limb-corrected SEVIRI Air Mass RGB. (c),(d) Insets of (a) and (b), respectively, showing only SEVIRI imagery centered over eastern Europe and the Mediterranean. The gray lines in all four panels indicate viewing zenith angles for SEVIRI greater than $75^{\circ}$. Color tile interpretation is as in Fig. 3 .

bands is sometimes similar depending on the vertical variation of water vapor in the atmosphere, such that taking the band difference effectively removes limb cooling from the red component of the RGB, even in the uncorrected imagery. In these cases, the limb correction algorithm naturally does not provide as much benefit to the red component as it does to the green and blue components.

\section{b. 1000 UTC 5 March 2016}

The second case study compares the 1000 UTC 5 March 2016 SEVIRI Air Mass RGB against the corresponding Aqua MODIS Air Mass RGB that passes near the eastern limb of the SEVIRI field of view, which includes the Arabian Peninsula. In Fig. 4a, limb-cooling causes stark differences between the uncorrected SEVIRI Air Mass RGB and the limb-corrected Aqua MODIS Air Mass RGB color interpretation. Additionally, the RGB distances for each color tile pair range from 35 to 65 due to large differences in each of the red, green, and blue components for each color tile pair. When the limb correction is applied for the SEVIRI Air Mass RGB (Fig. 4b), the color interpretation is more similar between SEVIRI and Aqua MODIS, with the color tile pairs showing visually similar colors, including the lower right color tile pair examining a region with high clouds. The limb-corrected RGB distances range from 10 to 41 , a sizable improvement over the RGB distances in the uncorrected imagery (Fig. 4a). Note that although the full SEVIRI disk is limb 

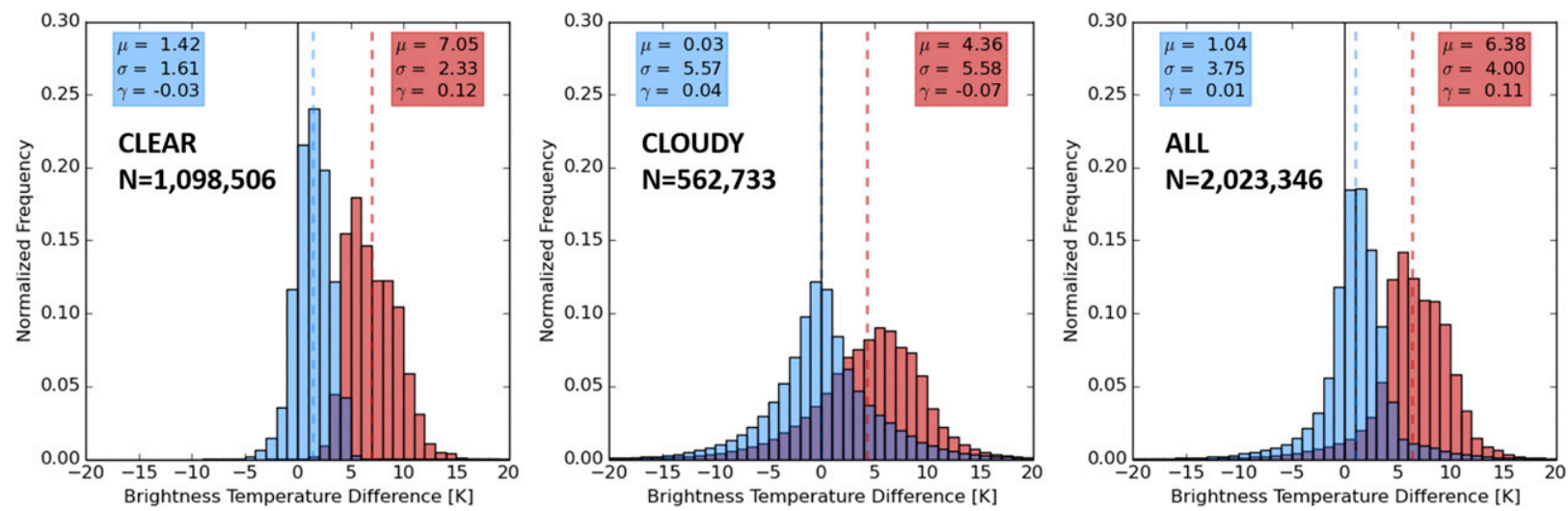

FIG. 5. Histograms corresponding to the case study shown in Fig. 4 representing departure of SEVIRI band 5 (6.2 $\mu \mathrm{m})$ brightness temperatures from the corresponding Aqua MODIS verification brightness temperatures (Aqua MODIS minus SEVIRI) for (left) clear pixels (SEVIRI and MODIS pixels that are cloud-free or with cloud-top pressures greater than $850 \mathrm{hPa}$ ), (center) cloudy pixels (SEVIRI and MODIS pixels with cloud-top pressures less than $850 \mathrm{hPa}$ ), and (right) all pixels (including pixels which are clear in Aqua MODIS and cloudy in SEVIRI, and vice versa). Only pixels with viewing zenith angles of $40^{\circ}-75^{\circ}$ are included in the histograms. Histograms are normalized by the total number of pixels $N$ in each category (i.e., clear, cloudy, all). The red histogram shows the distributions before limb correction, while the blue histogram shows the distributions after limb correction. Overlapping regions of the histograms are shown in purple. The mean $\mu$, standard deviation $\sigma$, and Yule-Kendall index $\gamma$ for each histogram are also listed with the mean of each distribution indicated by the vertical dotted lines.

corrected, the gray contour lines in Fig. 4 indicate viewing zenith angles greater than $75^{\circ}$, since the limb correction may not fully account for limb effects beyond this point.

To demonstrate the impact of limb correction on a continental scale, insets of the SEVIRI Air Mass RGB in Figs. $4 \mathrm{a}$ and $4 \mathrm{~b}$ are shown in Figs. $4 \mathrm{c}$ and $4 \mathrm{~d}$ centered over eastern Europe and the Mediterranean. The uncorrected SEVIRI Air Mass RGB (Fig. 4c) shows predominantly blue and purple coloring, although a stratospheric intrusion stretching from the Baltic states through eastern Europe toward the developing cyclone over Turkey is shown in red. While the uncorrected imagery provides useful information about the developing cyclone, the lack of contract between other air masses, such as those over eastern Europe and France, makes further interpretation difficult. The limb-corrected SEVIRI Air Mass RGB (Fig. 4d) shows greater contrast between features due to a greater contribution from the green component resulting from limb correction. The air mass over eastern Europe, for example, now shows a larger green component than the air mass over France, indicating that the air mass over eastern Europe has a higher tropopause height, and thus is a warmer air mass with higher moisture content. Low cloud features over Romania, Syria, France, and the United Kingdom are also more apparent. Furthermore, the thin cirrus clouds over central Ukraine and the Baltic states are more readily observed, since the blue coloring indicating the presence of thin cirrus due to the high moisture content no longer blends in with the surface, as was the case in the uncorrected imagery.
Figure 5 shows histograms of brightness temperature difference (Aqua MODIS minus SEVIRI) for SEVIRI band $5(6.2 \mu \mathrm{m})$ for clear pixels (SEVIRI and MODIS pixels that are cloud-free or with cloudtop pressures greater than $850 \mathrm{hPa}$ ), cloudy pixels (SEVIRI and MODIS pixels with cloud-top pressures less than $850 \mathrm{hPa}$ ), and all pixels (including pixels which are clear in Aqua MODIS and cloudy in SEVIRI, and vice versa), for viewing zenith angles from $40^{\circ}$ to $75^{\circ}$. Although pixels with cloud-top pressures greater than $850 \mathrm{hPa}$ may contain clouds, they are considered to be effectively clear since the optical pathlength is roughly equal to that of a clear pixel and complications due to parallax and observing the sides of these shallow clouds are minimized. In all three instances, the limb correction reduces the mean $\mu$ of the distribution to values of less than $1.5 \mathrm{~K}$. The standard deviation $\sigma$ for the clear pixels is also reduced. Although the limb-corrected cloudy pixel $\mu$ is almost $0 \mathrm{~K}, \sigma$ remains relatively unchanged, suggesting that the tails of the distributions capture the differences in the imagery caused by cloud movement between observation times of the two sensors. For Fig. 4, the time difference between equatorial samples is approximately $6 \mathrm{~min}$ (MODIS at 1002 UTC and SEVIRI at 1008 UTC). At Southern and Northern Hemisphere high latitudes, the time difference is approximately 15 and $5 \mathrm{~min}$, respectively.

Additionally, the two sensors are viewing clouds from different angles, resulting in parallax and differences in brightness temperature. The polar-orbiting sensor at a 
TABLE 3. Mean $\mu$, standard deviation $\sigma$, and Yule-Kendall index $\gamma$ of the Aqua MODIS minus SEVIRI brightness temperature difference corresponding to case shown in Fig. 4 for clear pixels only (SEVIRI and MODIS pixels that are cloud-free or with cloud-top pressures greater than $850 \mathrm{hPa}$ ), cloudy pixels only (SEVIRI and MODIS cloud-top pressures less than $850 \mathrm{hPa}$ ), and all pixels with viewing zenith angles of $40^{\circ}-75^{\circ}$.

\begin{tabular}{|c|c|c|c|c|c|c|c|}
\hline \multirow[b]{2}{*}{ Limb corrected? } & & \multicolumn{2}{|c|}{ Clear pixels } & \multicolumn{2}{|c|}{ Cloudy pixels } & \multicolumn{2}{|c|}{ All pixels } \\
\hline & & No & Yes & No & Yes & No & Yes \\
\hline \multirow[t]{3}{*}{ Band $5(6.2 \mu \mathrm{m})$} & $\mu$ & 7.05 & 1.42 & 4.36 & 0.03 & 6.38 & 1.04 \\
\hline & $\sigma$ & 2.33 & 1.61 & 5.58 & 5.57 & 4.00 & 3.75 \\
\hline & $\gamma$ & 0.12 & -0.03 & -0.07 & 0.04 & 0.11 & 0.01 \\
\hline \multirow[t]{3}{*}{ Band $6(7.3 \mu \mathrm{m})$} & $\mu$ & 6.55 & -0.65 & 5.66 & 2.23 & 6.56 & 0.54 \\
\hline & $\sigma$ & 2.29 & 1.24 & 8.13 & 8.65 & 5.29 & 5.71 \\
\hline & $\gamma$ & 0.19 & -0.14 & 0.03 & 0.37 & 0.21 & 0.09 \\
\hline \multirow[t]{3}{*}{ Band $7(8.7 \mu \mathrm{m})$} & $\mu$ & 3.96 & -0.77 & 5.28 & 4.36 & 4.93 & 1.67 \\
\hline & $\sigma$ & 3.10 & 2.67 & 13.45 & 13.56 & 8.84 & 9.28 \\
\hline & $\gamma$ & -0.08 & 0.10 & 0.17 & 0.20 & -0.01 & 0.26 \\
\hline \multirow[t]{3}{*}{ Band $8(9.6 \mu \mathrm{m})$} & $\mu$ & 12.60 & -0.43 & 8.46 & -3.09 & 11.70 & -0.86 \\
\hline & $\sigma$ & 4.08 & 2.18 & 8.64 & 8.41 & 6.64 & 5.96 \\
\hline & $\gamma$ & 0.01 & 0.09 & -0.10 & -0.14 & 0.03 & 0.05 \\
\hline \multirow[t]{3}{*}{ Band $9(10.8 \mu \mathrm{m})$} & $\mu$ & 3.60 & -0.48 & 4.38 & 4.07 & 4.43 & 1.87 \\
\hline & $\sigma$ & 3.07 & 2.98 & 14.08 & 14.16 & 9.36 & 9.80 \\
\hline & $\gamma$ & -0.07 & 0.02 & 0.25 & 0.26 & 0.04 & 0.17 \\
\hline \multirow[t]{3}{*}{ Band $10(12.0 \mu \mathrm{m})$} & $\mu$ & 4.21 & -0.37 & 4.67 & 4.37 & 4.94 & 2.07 \\
\hline & $\sigma$ & 3.27 & 3.16 & 13.72 & 13.82 & 9.28 & 9.79 \\
\hline & $\gamma$ & -0.07 & 0.08 & 0.29 & 0.29 & 0.06 & 0.19 \\
\hline
\end{tabular}

small viewing zenith angle may be observing the top of the cloud, whereas the geostationary sensor may be observing the side of the same cloud at a large viewing zenith angle. Thus, in this scenario, the polar-orbiting sensor is measuring the actual cloud-top temperature, whereas the geostationary sensor is measuring a brightness temperature with contributions from a $2-\mathrm{km}$ cloud layer lower in the atmosphere, which is likely to be warmer than the cloud-top. Cloud movement, parallax, or differences in observation angles are not addressed by the Elmer et al. (2016) limb correction algorithm. The Yule-Kendall index $\gamma$ (Wilks 2011), which is a robust and resistant measure of skewness, is also listed on the histograms. A positive $\gamma$ indicates that the distribution is skewed to the right, whereas a negative $\gamma$ points to a distribution skewed to the left.

The clear-pixel histogram in Fig. 5 represents the brightness temperature differences between Aqua MODIS and SEVIRI clear pixels shown in Fig. 4 that fall within the Aqua MODIS swath. Since SEVIRI viewing zenith angles are greater than Aqua MODIS viewing zenith angles across the entire swath, the Aqua MODIS minus SEVIRI brightness temperature difference is expected to be skewed to the right with a positive $\gamma$ in the uncorrected histograms, as observed. If limb effects are successfully removed from the imagery, a value of $\gamma$ close to zero in the limb-corrected histograms would result. We see that this is the case for both the clear-pixels-only histogram and the combined clearand-cloudy histogram.

In lieu of additional histograms, Table 3 lists the Aqua MODIS minus SEVIRI $\mu, \sigma$, and $\gamma$ sorted according to SEVIRI infrared bands 5-10, which include water vapor absorption bands (bands 5 and 6), an ozone absorption band (band 8), and infrared window bands (bands 7, 9, and 10). For all infrared bands, the distribution $\mu$ moves closer to $0 \mathrm{~K}$, although the greatest improvement is often seen when only clear pixels are considered. The value of $\sigma$ of the clear pixel distributions also decreases for all bands, but remains relatively unchanged for the cloudy pixel distributions. Most significantly, when both clear and cloudy pixels are considered, the $\mu$ decreases from an absolute difference of $4-12 \mathrm{~K}$ to an absolute difference of $0-2.1 \mathrm{~K}$ for all infrared bands. Although not shown in this paper, similar results were also observed for the previous case (Fig. 3).

\section{c. 1630 UTC 21 October 2016}

The third case study compares the 1630 UTC 21 October 2015 AHI Air Mass RGB against the corresponding Aqua MODIS Air Mass RGB that passes through the center of the AHI field of view (Fig. 6), similar to the first case study for SEVIRI. In this case, 

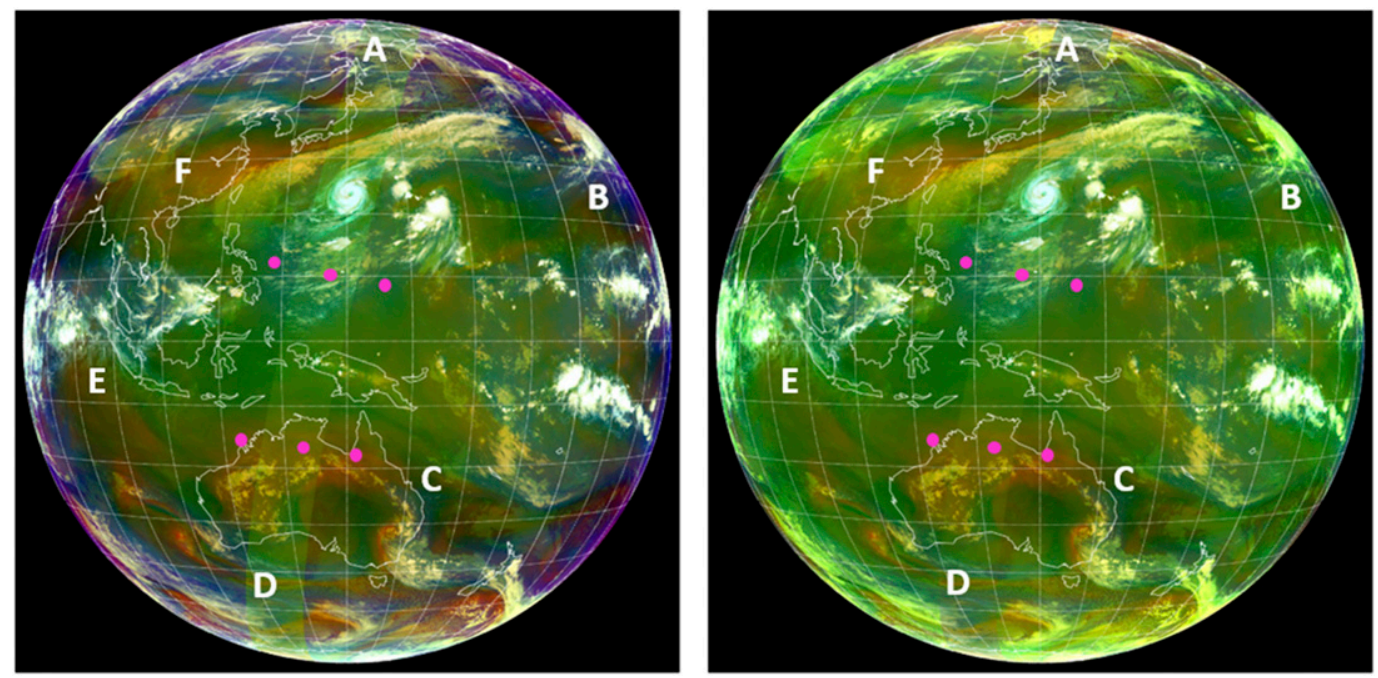

FIG. 6. The 1630 UTC 21 October 2015 limb-corrected Aqua MODIS Air Mass RGB (viewing zenith angles $<40^{\circ}$ only) overlaying the corresponding (a) uncorrected and (b) limb-corrected AHI Air Mass RGB. White letters increasing clockwise indicate the location of Aqua AIRS and NUCAPS atmospheric profiles shown in Fig. 8. Parallels and meridians are shown for every $10^{\circ}$ of latitude and longitude, respectively, with $\left(0^{\circ}, 140^{\circ} \mathrm{E}\right)$ in the center of the image. This example is also shown in Elmer et al. (2016); the magenta dots indicate the location of the Aqua AIRS atmospheric profiles from that paper for this same case for comparison.

both the AHI and MODIS Air Mass RGB recipes were adjusted using the Berndt et al. (2018) methodology to mimic the SEVIRI RGB recipe. This case in Fig. 6 was also included in Elmer et al. (2016), but the AHI imagery was not limb-corrected for that study and thus the full AHI image was not shown. Note that the AHI cloud product was not obtainable for this case, so the cloud correction was not performed (thus, $Q=1.0$ for all pixels during limb correction). Prior to limb correction of AHI (Fig. 6a), limb cooling is evident around the edges of the full disk, with the limb-corrected Aqua MODIS swath easily identifiable. At high latitudes, the uncorrected AHI Air Mass RGB is showing a smaller green contribution than the nearby Aqua MODIS Air Mass RGB. After the limb correction of the AHI Air Mass RGB (Fig. 6b), the Aqua MODIS and AHI Air Mass RGBs closely resemble each other, with more similarity in the red, green, and blue coloring in many locations along the edge of the Aqua MODIS swath shown.

Histograms of the brightness temperature difference (Аqua MODIS minus AHI) for all four infrared bands used in the Air Mass RGB are shown in Fig. 7. The histograms include both clear and cloudy pixels. Similar to the results show in Table 3 for SEVIRI, the limb correction reduces $\mu$ for all four infrared bands shown from an absolute difference of approximately $2-8 \mathrm{~K}$ to within an absolute difference of $0-1.1 \mathrm{~K}$. Note that the largest shifts occur for the water vapor and ozone absorption bands (bands 8, 10, and 12). The "clean" infrared window band (band 13), which is mostly free of atmospheric absorption, is not greatly affected by limb effects. Therefore, the limb correction is not as significant for this band (reducing the mean brightness temperature difference only $1 \mathrm{~K}$ from 1.87 to $0.87 \mathrm{~K}$ ).

At high viewing angles, limb effects can make it difficult to discern the horizontal extent of polar air masses at high latitudes and interfere with the interpretation of tropical air masses at low latitudes. Six satellite-derived atmospheric profiles are shown in Fig. 8 which correspond to the locations indicated by the white letters in Fig. 6 . The locations of these profiles were chosen in order to highlight different atmospheric features within the AHI Air Mass RGB across all latitudes and varying viewing zenith angles. These profiles provide insight into the atmospheric conditions regarding temperature, moisture, and tropopause height, enabling the color interpretation of the limb-corrected Air Mass RGB to be verified against observations.

Profile A (Fig. 8a) shows cold upper-level temperatures of less than $-50^{\circ} \mathrm{C}$ with extremely dry midand upper levels. The dry upper-level conditions in the profile are consistent with the red/orange coloring in the MODIS overlay (Fig. 6), whereas the purple coloring indicates a cold, polar air mass. There is no green contribution, as the tropopause is low, near $375 \mathrm{hPa}$. 

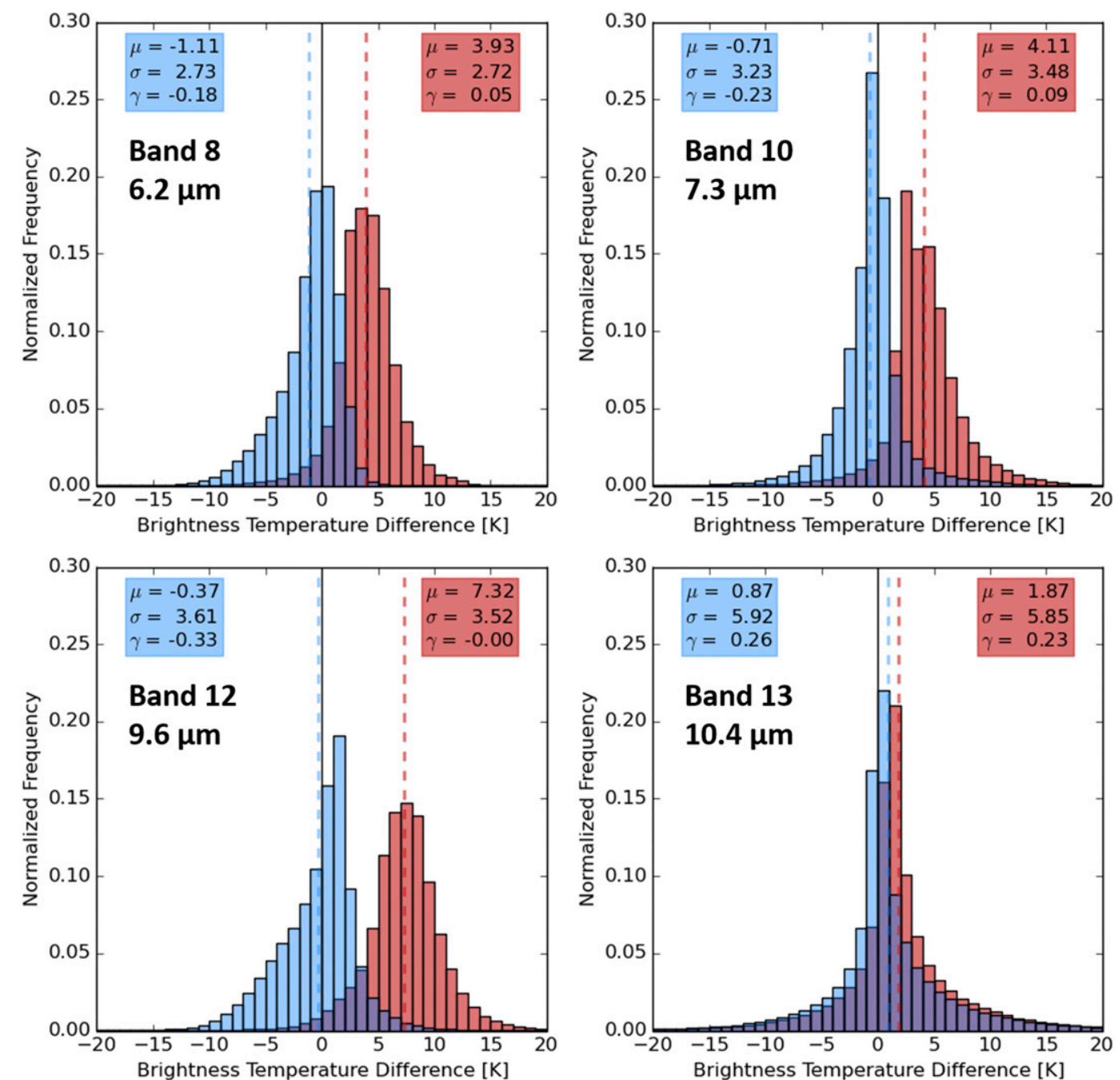

FIG. 7. Histograms corresponding to the case shown in Fig. 6 representing departure of AHI brightness temperatures from the corresponding Aqua MODIS verification brightness temperatures (Aqua MODIS minus AHI) before (red) and after (blue) limb correction for AHI bands (top left) $8(6.2 \mu \mathrm{m})$, (top right) $10(7.3 \mu \mathrm{m})$, (bottom left) $12(9.6 \mu \mathrm{m})$, and (bottom right) $13(10.4 \mu \mathrm{m})$. Overlapping regions of the histograms are shown in purple. Histograms are normalized based on the total number of pixels (1994285) including both clear and cloudy pixels with viewing zenith angles of $40^{\circ}-75^{\circ}$. The mean $\mu$, standard deviation $\sigma$, and Yule-Kendall index $\gamma$ for each histogram are also listed with the mean of each distribution indicated by the vertical dotted lines.

Profile B (Fig. 8b) is located in the tropics at a relatively high satellite zenith angle. Here, limb effects can cause false blue coloring of warm, moist tropical air masses, which should be green in coloring. If polar air were indeed present, one would expect upper-level temperatures much colder than the $-30^{\circ} \mathrm{C}$ at $300 \mathrm{hPa}$ observed in profile B (Fig. 8b). In addition, the profile captures a moist layer above $300 \mathrm{hPa}$ with a smaller temperature and dewpoint depression which is consistent with presence of a warm, moist upper-level air mass colored green in the limb-corrected RGB (Fig. 6b). Thus, the limb correction allows for more accurate diagnosis of tropical air masses that are important for assessing the influence of moisture plumes on the development and intensification of tropical cyclones (Kaplan and DeMaria 2003; Vizy and Cook 2010).

In contrast to profile $\mathrm{B}$, profile $\mathrm{C}$ (Fig. 8c) is in a warm, tropical air mass with more upper-level dry air present. At this location, limb effects are smaller than at profile $\mathrm{B}$, so the colors in the uncorrected and limbcorrected images are similar (Fig. 6). The profile does confirm dry conditions aloft, correctly interpreted from the orange in the AHI Air Mass RGB (Fig. 6), and an average tropopause height which contributes to the green component. 

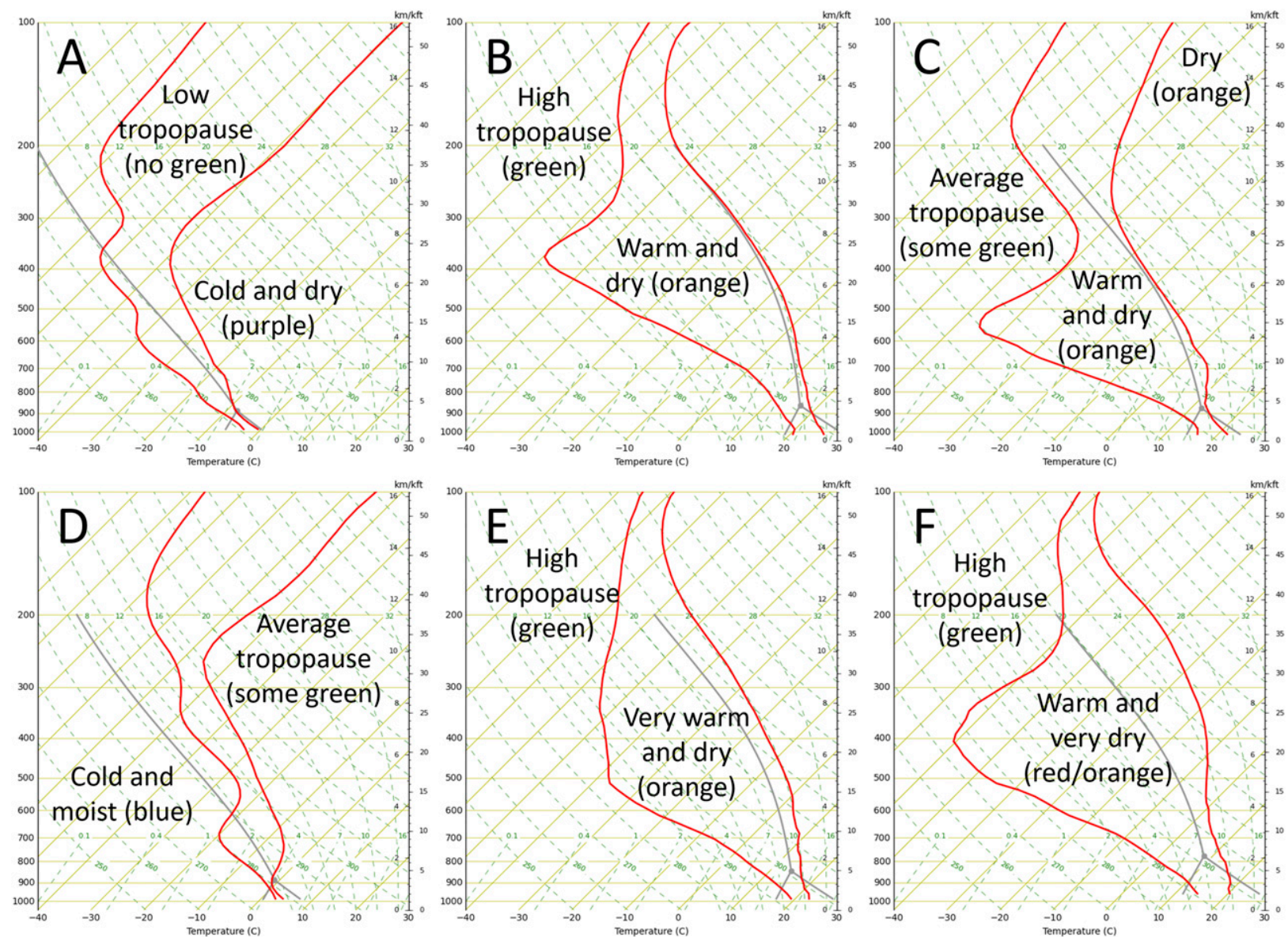

FIG. 8. The 21 October 2015 Aqua AIRS and NUCAPS profiles of temperature and dewpoint associated with the air mass below the corresponding white letters in Fig. 6. (a) 1615 UTC Aqua AIRS profile at $50^{\circ} \mathrm{N}, 145^{\circ} \mathrm{E}$; (b) 1326 UTC NUCAPS profile at $20^{\circ} \mathrm{N}$, $170^{\circ} \mathrm{W}$; (c) $1520 \mathrm{UTC}$ NUCAPS profile at $25^{\circ} \mathrm{S}, 155^{\circ} \mathrm{E}$; (d) $1640 \mathrm{UTC}$ Aqua AIRS profile at $50^{\circ} \mathrm{S}, 120^{\circ} \mathrm{E}$; (e) $1840 \mathrm{UTC}$ NUCAPS profile at $10^{\circ} \mathrm{S}, 95^{\circ} \mathrm{E}$; and (f) $1829 \mathrm{UTC}$ NUCAPS profile at $25^{\circ} \mathrm{N}, 110^{\circ} \mathrm{E}$.

At profile D (Fig. 8d), cold temperatures on the order of $-50^{\circ} \mathrm{C}$ are observed aloft with an increasing dewpoint depression near the 300-hPa level with a moister atmospheric column below, which yields both a blue and red contribution to the Air Mass RGB (Fig. 6). As inferred from the air mass in the AHI imagery closest to profile D (Fig. 6), the limb correction adds a green contribution, indicating that the tropopause is higher than near profile $\mathrm{A}$, where a minimal green contribution is observed after limb correction.

At profile $\mathrm{E}$ in Fig. 6, the olive coloring represents a warm, dry tropical air mass, which is confirmed by the warm temperatures and large dewpoint depressions in the 500-200-hPa layer in profile E (Fig. 8e). In the uncorrected Air Mass RGB (Fig. 6a), the orange colors are more intense than in the limb-corrected Air Mass RGB, erroneously suggesting a very dry upperlevel and low tropopause. However the olive coloring (a combination of orange and green components) in the limb-corrected Air Mass RGB suggests that the tropopause is fairly high, which is confirmed by profile $\mathrm{E}$ (Fig. 8e) as being near $150 \mathrm{hPa}$. Profiles E and F look similar, except that profile $\mathrm{F}$ (Fig. 8f) is drier in the 300-500-hPa layer. In the uncorrected Air Mass RGB (Fig. 6a) at points $\mathrm{E}$ and $\mathrm{F}$, the orange coloring indicative of dry air is similar. However, the limbcorrected Air Mass RGB rightly shows that point $\mathrm{F}$ is much drier than point $\mathrm{E}$, since the orange coloring is more intense at point $\mathrm{F}$. As shown by comparing profiles $\mathrm{E}$ and $\mathrm{F}$, the advantage of the limb-corrected Air Mass RGB is that it enhances the ability to distinguish between dry and extremely dry regions where the depth or water vapor content of the dry air differs.

\section{d. 0000 UTC 6 September 2017}

The fourth case study, depicting Hurricanes Irma and Jose from 0000 UTC 6 September 2017, focuses on the overlapping regions of GOES-16 $\mathrm{ABI}$ and SEVIRI, 
which lies roughly between $65^{\circ}$ and $25^{\circ} \mathrm{W}$ at the time the imagery was collected (see Fig. 2). In this region, the western limb of SEVIRI overlaps the near-nadir points of GOES-16 ABI and the eastern limb of GOES16 ABI overlaps the near-nadir points of SEVIRI. The GOES-16 uncorrected and limb-corrected Air Mass RGB composites are shown side by side in Figs. 9a and $9 \mathrm{~b}$, respectively, whereas the SEVIRI uncorrected and limb-corrected Air Mass RGB composites are shown in Figs. 9c and 9d, respectively. Qualitatively, the limb-corrected RGB composites present a more consistent interpretation between the two sensors than the uncorrected RGB composites in the overlapping regions. Figure 9e splices together the uncorrected ABI and SEVIRI Air Mass RGBs (Figs. 9a,c) by taking the ABI or SEVIRI pixel with the smallest viewing zenith angle. In this case, all points west of $44.75^{\circ} \mathrm{W}$ are contributed by $\mathrm{ABI}$, whereas all points east of $44.75^{\circ} \mathrm{W}$ are contributed by SEVIRI. Figure $9 \mathrm{f}$ is the limbcorrected view of Fig. 9e. Since the limb correction corrects the infrared imagery so that all brightness temperatures are adjusted to mimic a nadir view, the North Atlantic in the multisensor limb-corrected Air Mass RGB (Fig. 9f) can be correctly interpreted even though the viewing zenith angles for both sensors are greater than $40^{\circ}$. The consistent coloring and interpretation allow for increased efficiency in tracking synopticscale moist and dry air masses that impact development, intensification, and weakening of midlatitude cyclones and hurricanes.

\section{Summary and conclusions}

SEVIRI, AHI, ABI, and Aqua MODIS infrared channels were limb-corrected using sensor-specific limb correction coefficients, but they depict similar limb-corrected brightness temperatures and resulting RGB interpretation for all viewing zenith angles. These results indicate that the Elmer et al. (2016) limb correction technique effectively removes limb effects from infrared imagery for geostationary sensors as well as polar-orbiting sensors, enabling improved interpretation of RGB composites. The intercalibration technique of Berndt et al. (2018) also performs well by supporting consistent interpretation of RGB composites derived from different sensors. Furthermore, the removal of limb effects and intercalibration is shown to support the simultaneous analysis of atmospheric features from multiple sensors and enables the creation of mosaicked RGB composites with global coverage and standardized interpretation to enhance tracking of synoptic-scale atmospheric features.
Limb-corrected RGB composites enable atmospheric features on the limb to be correctly interpreted with high confidence, since they enable more accurate interpretation of atmospheric features due to improved RGB coloring. As a result, operational forecasters will have improved situational awareness and can produce more informed forecasts. Additionally, limb correction leads to more consistent imagery, such that air masses with similar characteristics will have the same coloring regardless of whether they are on the limb or at nadir. This improves the spatial coverage of accurate RGB imagery. The limb correction is also not computationally intensive for geostationary sensors. As with polar orbiters, the limiting factor is the latency of the cloud product for the purpose of accounting for cloud effects. However, as noted by Elmer et al. (2016) and demonstrated by the third case study comparing Aqua MODIS and AHI Air Mass RGBs, correcting only for limb effects while assuming a full optical pathlength $(Q=1)$ still provides a significant improvement in RGB interpretation.

Note that after the limb correction of Air Mass RGBs, much of the blue coloring at the poles is obscured by an increase in green coloring. The increased representation of the influence of the green component at high viewing angles improves the coloring and interpretation of features important for identifying stratospheric intrusions to anticipate changes in midlatitude cyclone intensity and increases the ability to track moisture plumes and dry layers that are integral to anticipating hurricane intensity changes. The original EUMETSAT Air Mass RGB recipe was tuned specifically for the midlatitudes using uncorrected imagery. Although the limb correction improves the imagery by removing anomalous cooling and false coloring, it also reduces color contrasts on the limb that many forecasters are accustomed to. Thus, the RGB recipe may need to be retuned to get optimal color contrasts, but not too much to return it to its original, pre-limb-correction coloring.

The Elmer et al. (2016) limb correction algorithm has been tested in real-time for MODIS and VIIRS RGB composites produced at the NASA Short-term Prediction Research and Transition (SPoRT) Center, and has been successfully evaluated by operational end users at National Oceanic and Atmospheric Administration (NOAA) National Weather Service (NWS) Weather Forecast Offices (WFOs) and National Centers (Fuell et al. 2016; Berndt et al. 2017). As a result, the Elmer et al. (2016) limb correction algorithm is currently being applied to real-time geostationary imagery by NASA SPoRT, making limb-corrected RGB composites available within the Advanced Weather Interactive Processing System, version 2 (AWIPS-2), and the 

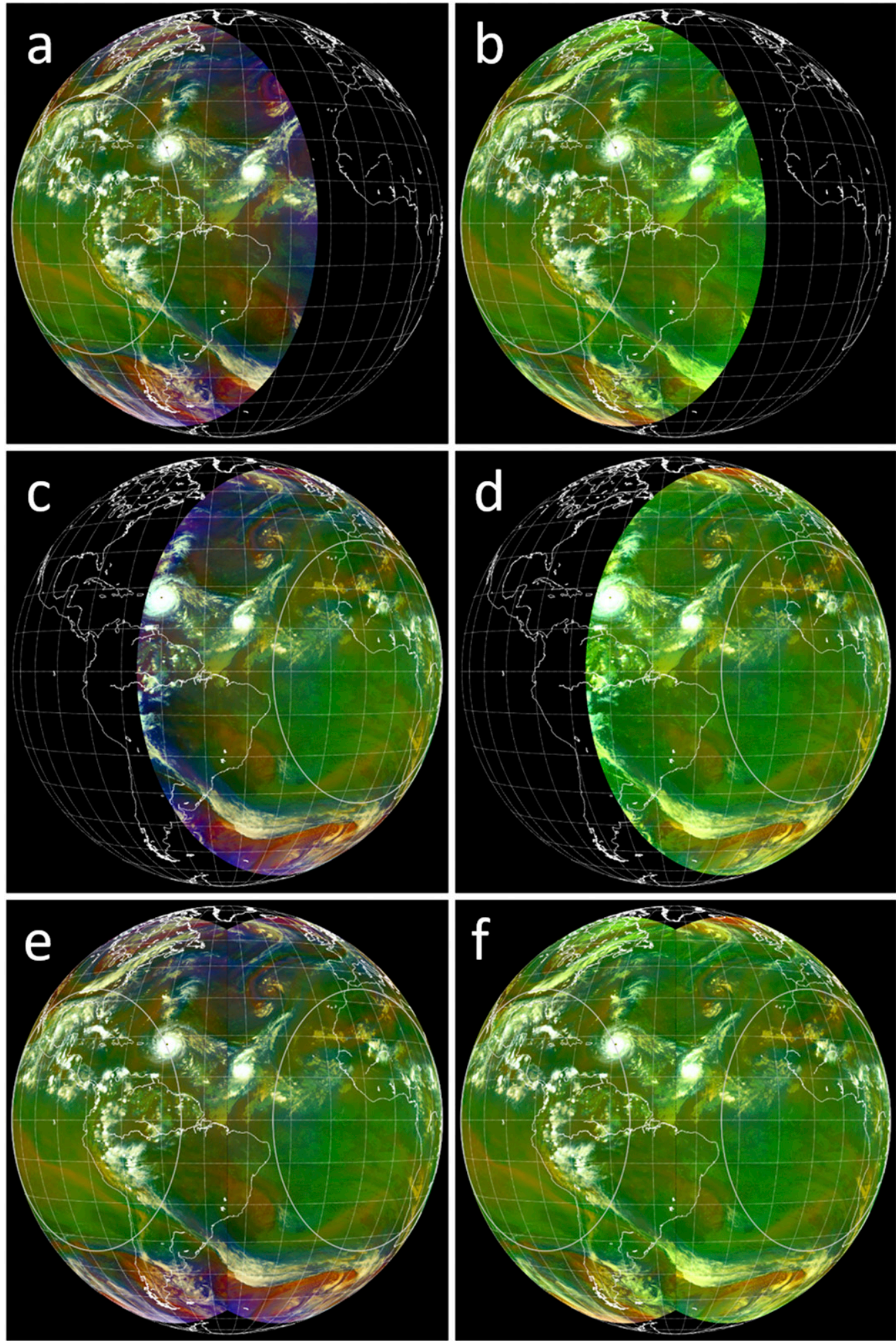

FIG. 9. The 0000 UTC 6 September 2017 (a) uncorrected ABI, (b) limb-corrected ABI, (c) uncorrected SEVIRI, and (d) limb-corrected SEVIRI Air Mass RGBs. (e) A splice of the left half of (a) and the right half of (c), and (f) a splice of the left half of (b) and the right half of (d). The gray contour line indicates a viewing zenith angle of $40^{\circ}$ and the pixels with viewing zenith angles beyond $75^{\circ}$ have been removed. 
NCEP Advanced Weather Interactive Processing System (N-AWIPS) for initial testing and evaluation of limb-corrected geostationary Air Mass RGBs in the operational environment at national centers.

Acknowledgments. The authors thank Kevin McGrath and Paul Meyer for assistance obtaining and processing the GOES-16 ABI imagery. Frank LaFontaine and Matt Smith assisted in transitioning the limb correction algorithm into operations.

\section{REFERENCES}

Aumann, H. H., and Coauthors, 2003: AIRS/AMSU/ HSB on the Aqua mission: Design, science objectives, data products, and processing systems. IEEE Trans. Geosci. Remote Sens., 41, 253-264, https://doi.org/ 10.1109/TGRS.2002.808356.

Berndt, E. B., B. T. Zavodsky, and M. J. Folmer, 2016: Development and application of Atmospheric Infrared Sounder ozone retrieval products for operational meteorology. IEEE Trans. Geosci. Remote Sens., 54, 958-967, https://doi.org/10.1109/ TGRS.2015.2471259.

_ A. Molthan, W. W. Vaughan, and K. Fuell, 2017: Transforming satellite data into weather forecasts, Eos, 98, https:// doi.org/10.1029/2017EO064449.

Berndt, E., N. Elmer, L. Schultz, and A. Molthan, 2018: A methodology to determine recipe adjustments for multispectral composites derived from next-generation advanced satellite imagers. J. Atmos. Oceanic Technol., 35, 643-664, https:// doi.org/10.1175/JTECH-D-17-0047.1.

Elmer, N. J., E. Berndt, and G. Jedlovec, 2016: Limb correction of MODIS and VIIRS infrared channels for the improved interpretation of RGB composites. J. Atmos. Oceanic Technol., 33, 1073-1087, https://doi.org/10.1175/ JTECH-D-15-0245.1.

EUMETSAT, 2015a: Airmass RGB. EUMETSAT, http:// oiswww.eumetsat.int/ idds/html/doc/airmass_interpretation.pdf.

—, 2015b: EUMETSAT Data Centre. EUMETSAT, http:// www.eumetsat.int/website/home/Data/DataDelivery/ EUMETSATDataCentre/index.html.

_ 2018: Meteosat Third Generation. EUMETSAT, http:// www.eumetsat.int/website/home/Satellites/FutureSatellites/ MeteosatThirdGeneration/index.html.

EUMETSAT User Services, 2009: Best practices for RGB compositing of multi-spectral imagery. EUMETSAT, 8 pp., http:// oiswww.eumetsat.int $/ \sim$ idds $/ \mathrm{html} /$ doc/best_practices.pdf.

Fuell, K. K., B. J. Guyer, D. Kann, A. L. Molthan, and N. Elmer, 2016: Next generation satellite RGB dust imagery leads to operational changes at NWS Albuquerque. J. Oper. Meteor., 4, 75-91, https://doi.org/10.15191/nwajom.2016.0406.

Gambacorta, A., 2013: The NOAA Unique CrIS/ATMS Processing System (NUCAPS). Algorithm Theoretical Basis Doc, version 1.0, NOAA, 73 pp., www.ospo.noaa.gov/ Products/atmosphere/soundings/nucaps/docs/NUCAPS_ATBD_ 20130821.pdf.

- - and C. D. Barnet, 2013: Methodology and information content of the NOAA NESDIS operational channel selection for the Cross-Track Infrared Sounder (CrIS). IEEE Trans. Geosci. Remote Sens., 51, 3207-3216, https://doi.org/ 10.1109/TGRS.2012.2220369.
Goldberg, M. D., D. S. Crosby, and L. Zhou, 2001: The limb adjustment of AMSU-A observations: Methodology and validation. J. Appl. Meteor., 40, 70-83, https://doi.org/10.1175/ 1520-0450(2001)040<0070:TLAOAA $>2.0 . \mathrm{CO} ; 2$.

GSFC, 2015: LAADS Web: Level 1 and Atmosphere Archive and Distribution System. NASA, https://ladsweb.modaps.eosdis.nasa.gov/.

Han, Y., P. van Delst, Q. Liu, F. Weng, B. Yan, R. Treadon, and J. Derber, 2006: JCSDA Community Radiative Transfer Model (CRTM)-Version 1. NOAA Tech. Rep. NESDIS 122, 33 pp., http://www.star.nesdis.noaa.gov/sod/sst/micros/pdf/ CRTM_v1_NOAAtechReport-1.pdf.

Hidehiki, M., T. Masaya, K. Yuki, 2015: VIS and IR bands of Himawari-8/AHI compatible with those of MTSAT-2/Imager. Japan Meteorological Agency Meteorological Satellite Center Tech. Note 60, 18 pp., http://www.data.jma.go.jp/mscweb/en/ product/library/note/index.html.

Kaplan, J., and M. DeMaria, 2003: Large-scale characteristics of rapidly intensifying tropical cyclones in the North Atlantic basin. Wea. Forecasting, 18, 1093-1108, https://doi.org/10.1175/ 1520-0434(2003)018<1093:LCORIT>2.0.CO;2.

Kerkmann, J., 2016: MTG training plans and rapid scan experiments/training workshop. 2016 NOAA Satellite Proving Ground/User-Readiness Meeting, Norman, OK, NOAA, https://courses.comet.ucar.edu/mod/resource/view.php?id= $10834 \&$ forceview $=1$.

Lensky, I. M., and D. Rosenfeld, 2008: Clouds-AerosolsPrecipitation Satellite Analysis Tool (CAPSAT). Atmos. Chem. Phys., 8, 6739-6753, https://doi.org/10.5194/acp-8-6739-2008.

Lindsey, D. T., T. J. Schmit, W. M. MacKenzie Jr., C. P. Jewett, M. M. Gunshor, and L. Grasso, 2012: $10.35 \mu \mathrm{m}$ : Atmospheric window on the GOES-R Advanced Baseline Imager with less moisture attenuation. J. Appl. Remote Sens., 6, 063598, https:// doi.org/10.1117/1.JRS.6.063598.

Nalli, N. R., and Coauthors, 2016: Satellite sounder observations of contrasting tropospheric moisture transport regimes: Saharan air layers, Hadley cells, and atmospheric rivers. J. Hydrometeor., 17, 2997-3006, https://doi.org/ 10.1175/JHM-D-16-0163.1.

- , and Coauthors, 2018: Validation of atmospheric profile retrievals from the SNPP NOAA-Unique Combined Atmospheric Processing System. Part 1: Temperature and moisture. IEEE Trans. Geosci. Remote Sens., 56, 180-190, https:// doi.org/10.1109/TGRS.2017.2744558.

Scarino, B. R., D. R. Doelling, P. Minnis, A. Gopalan, T. Chee, R. Bhatt, C. Lukashin, and C. O. Haney, 2016: A web-based tool for calculating spectral band difference adjustment factors derived from SCIAMACHY hyperspectral data. IEEE Trans. Geosci. Remote Sens., 54, 2529-2542, https://doi.org/ 10.1109/TGRS.2015.2502904.

Schmetz, J., P. Pili, S. Tjemkes, D. Just, J. Kerkmann, S. Rota, and A. Ratier, 2002: An introduction to Meteosat Second Generation (MSG). Bull. Amer. Meteor. Soc., 83, 977-992, https://doi.org/10.1175/1520-0477(2002)083<0977:AITMSG> 2.3. $\mathrm{CO} ; 2$

Schmit, T. J., M. M. Gunshor, W. P. Menzel, J. J. Gurka, J. Li, and A. S. Bachmeier, 2005: Introducing the next-generation Advanced Baseline Imager on GOES-R. Bull. Amer. Meteor. Soc., 86, 1079-1096, https://doi.org/10.1175/BAMS-86-8-1079.

Shimizu, A., 2015: Introduction of JMA VLab Support Site on RGB Composite Imagery and tentative RGBs. Sixth Asia/Oceania Meteorological Satellite Users' Conference, Tokyo, Japan, Japan Meteorological Agency, http://www.data.jma.go.jp/mscweb/en/ aomsuc6_data/presentations.html. 
Soden, B. J., and F. P. Bretherton, 1993: Upper tropospheric relative humidity from the GOES $6.7 \mu \mathrm{m}$ channel: Method and climatology for July 1987. J. Geophys. Res., 98, 16 669-16 688, https://doi.org/10.1029/93JD01283.

Vizy, E. K., and K. H. Cook, 2010: Influence of the Amazon/Orinoco Plume on the summertime Atlantic climate. J. Geophys. Res., 115, D21112, https://doi.org/10.1029/2010JD014049.

Wilks, D. S., 2011: Statistical Methods in the Atmospheric Sciences. 3rd ed. International Geophysics Series, Vol. 100, Academic Press, 704 pp.
Zavodsky, B. T., A. L. Molthan, and M. J. Folmer, 2013: Multispectral imagery for detecting stratospheric air intrusions associated with mid-latitude cyclones. J. Oper. Meteor., 1, 71-83, https://doi.org/10.15191/nwajom.2013.0107.

Zeschke, B., 2015: Himawari-8 derived RGB products applied to the Australasia-Pacific region. Sixth Asia/Oceania Meteorological Satellite Users' Conference, Tokyo, Japan, Japan Meteorological Agency, http://www.data.jma.go.jp/mscweb/en/ aomsuc6_data/presentations.html. 